\title{
Sex Steroids and Breast Cancer: An Overview*
}

\author{
Zeina Nahleh $^{1 \#}$, Juan E. Arenas ${ }^{2}$, Arafat Tfayli ${ }^{3}$ \\ ${ }^{1}$ Department of Internal Medicine, Division of Hematology-Oncology, Paul L. Foster School of Medicine, Texas Tech University \\ Health Sciences Center, El Paso, USA; ${ }^{2}$ Department of Internal Medicine, Paul L. Foster School of Medicine, Texas Tech University \\ Health Sciences Center, El Paso, USA; ${ }^{3}$ Department of Internal Medicine, American University of Beirut Medical Center, Beirut, \\ Lebanon. \\ Email: " zeina.nahleh@ttuhsc.edu, juan.arenas@ttuhsc.edu, at35@aub.edu.lb
}

Received April $4^{\text {th }}, 2013$; revised May $3^{\text {rd }}, 2013$; accepted May $11^{\text {th }}, 2013$

Copyright (C) 2013 Zeina Nahleh et al. This is an open access article distributed under the Creative Commons Attribution License, which permits unrestricted use, distribution, and reproduction in any medium, provided the original work is properly cited.

\begin{abstract}
Sex steroids, also known as sex hormones are synthesized naturally by the gonads (ovaries or testes). The two main classes of sex steroids, androgens and estrogens, are crucial hormones for the proper development and function of the body; they regulate sexual differentiation, the secondary sex characteristics, and sexual habits. They are also well known to influence many common cancers, especially hormonally driven cancers such as breast and prostate cancer. In this report, we review the association of sex steroids with cancer and the potential use of endocrine manipulation in cancer therapy as well as the limitations and challenges faced in this field.
\end{abstract}

Keywords: Sex Steroids; Hormones; Breast; Cancer

\section{Introduction}

Sex steroids, also known as sex hormones are synthesized naturally by the gonads (ovaries or testes) [1], and are released into the blood to reach target tissues and exert their endocrine action, or by conversion from other sex steroids in other tissue such as liver or fat. The two main classes of sex steroids are androgens and estrogens, of which the most important human derivatives are testosterone and estradiol, respectively. Androgens also include androstenedione, dehydroepiandrosterone, and dihydrotestosterone. Estrogens include estriol and estrone [2]. Progesterone is a third class of sex steroids, distinct from androgens and estrogens. Progesterone is the most important and only naturally occurring human progestogen. In general, androgens are considered "male sex hormones" since they have masculinizing effects, while estrogens and progestogens are considered "female sex hormones" although all types are present in each sex, albeit at different levels (Figures 1 and 2). In this report, we review the association of sex steroids with cancer and the potential use of endocrine manipulation in cancer therapy as well as the limitations and challenges faced in this field.

\footnotetext{
*All authors report no conflict of interest.

\#Corresponding author.
}

\section{Sex Steroids and Risk for Cancer}

Sex steroids are crucial hormones for the proper development and function of the body; they regulate sexual differentiation, the secondary sex characteristics, and sexual habits. They are also well known to influence many common cancers [3], most notably the 2 hormonally related cancers: breast [4,5] and prostate cancer [6] due to interplay between a variety of hormonally related and genetic risk factors. As an example, high levels of circulating testosterone and low levels of sex hormonebinding globulin (SHBG) are associated with increased risks of prostate cancer. Low levels of circulating estradiol may represent an additional risk factor [6]. Breast cancer risk for postmenopausal women is positively associated with the circulating concentrations of estrogens and androgens [7-9]. Also, it has been suggested that the increase in risk of postmenopausal breast cancer associated with obesity may be largely explained by the relatively high circulating concentrations of free estradiol in obese postmenopausal women $[10,11]$.

\section{Issues with Understanding the Role of Sex Steroids in Cancer}

Despite the relatively extensive research and epidemiological studies examining the correlation of sex steroids and the risk of breast cancer, several potential limitations 
Sex Steroids Production in women

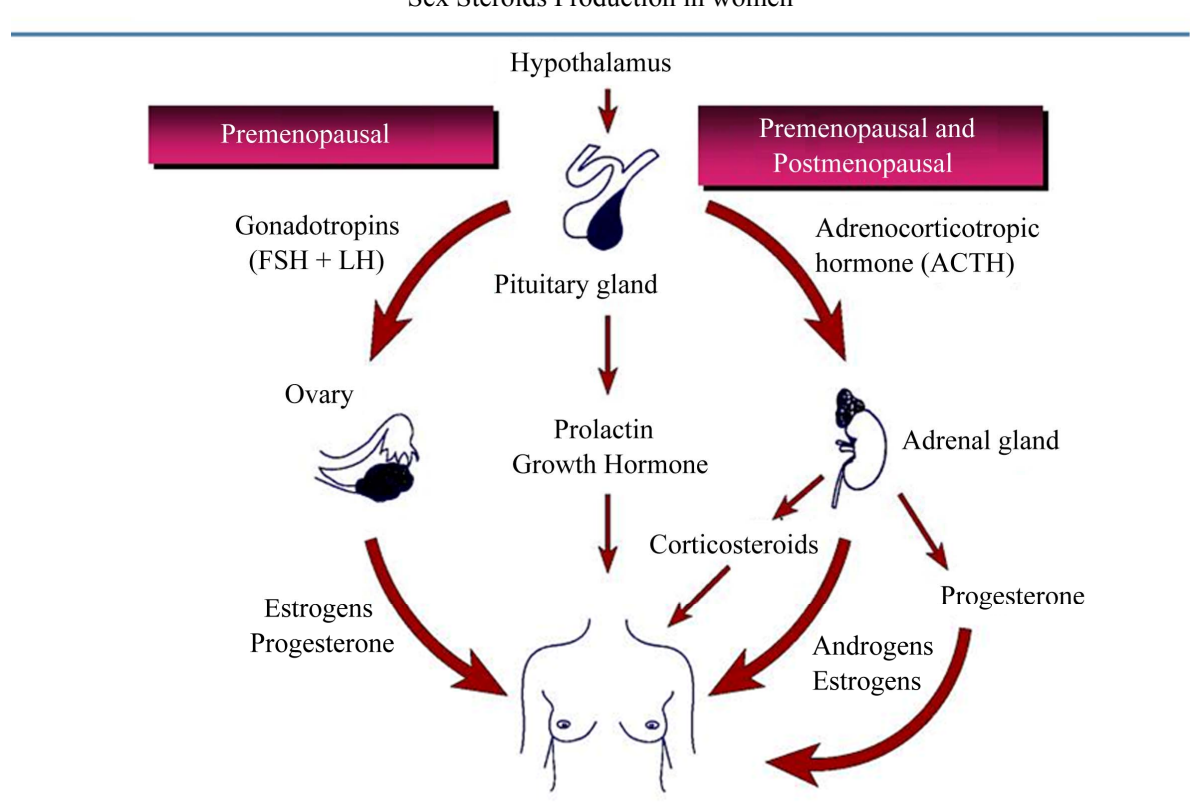

Figure 1. Sex hormones production in women.

Sex Steroids Production in Men

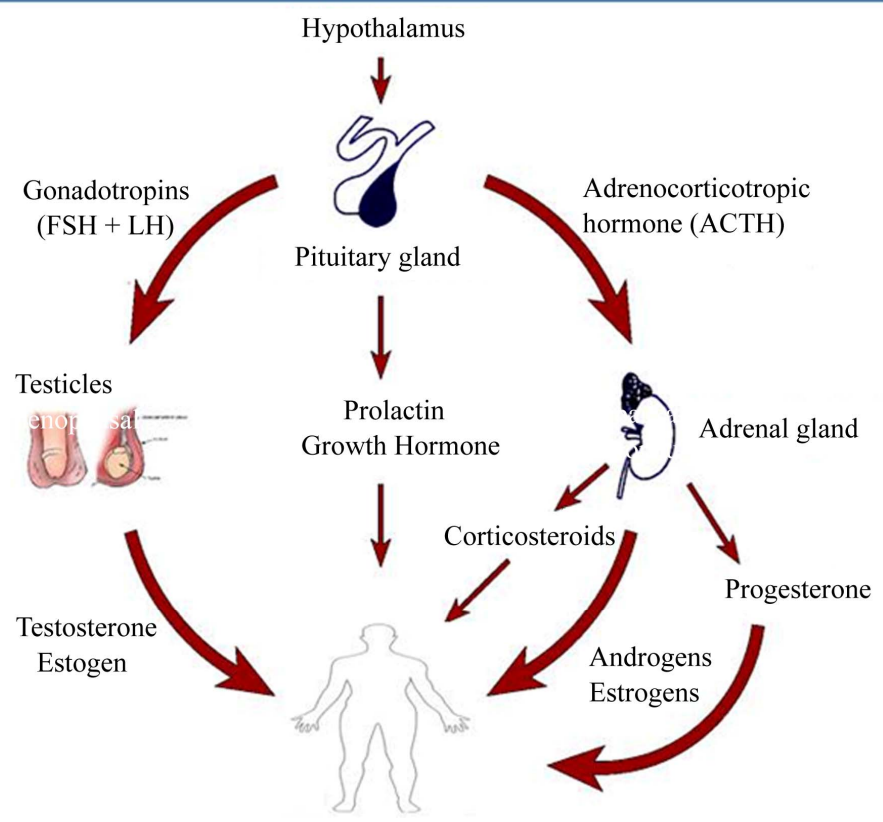

Figure 2. Sex hormones production in men.

have lead to failing in fully understanding the role of endogenous hormones in cancer etiology. These can be summarized as follows: 1) difficulties in reliably measuring sex hormones, which are complex in both their metabolic pathways and interactions with other biological markers [12-14]. This is especially true for premenopausal women, whereby the wide variation in hormonal levels over the duration of the menstrual cycle requires careful attention to the timing of sample collections.
Similarly, serum testosterone and androstenedione levels are the most commonly measured androgens, but they also demonstrate substantial daily variability, while most epidemiological data are based on a single blood sample collected at nonstandard times [14]; 2) the lack of reliability of serum steroid levels measured by radioimmunoassay. The appropriate methods to reliably measure sex hormones remain unclear. Radioimmunoassays have been most commonly used to measure estrogens, how- 
ever, other sensitive methods are being evaluated, including a liquid chromatography-mass spectrometry technique that simultaneously measures 15 estrogen metabolites [15]. Future studies are needed to better understand interrelationships of endogenous hormones with other circulating markers associated with breast cancer risk, including androgens, as well as isoforms relevant to breast cancer. A recent novel approach for measuring estrogens was reported using urinary estrone glucuronide (E1G) in premenopausal women [16]. The method accounts for cyclic variation in hormone levels during the periovulatory and luteal phases of the menstrual cycle. Using that approach, Johnson et al. [17] observed that lower urinary E1G levels were associated with a single-nucleotide polymorphism (SNP) in the cytochrome P450 3A gene (CYP3A). Furthermore, using data from targeted genotyping and genome-wide association study (GWAS) datasets comprising more than 10,000 breast cancers and 17,000 control subjects, a proxy SNP that tags the CYP3A SNP was found to be associated with a weak reduction in the risk of breast cancer, notably in women younger than 50 years. This finding could reflect an enhanced ability to detect gene-hormone interactions among women with higher estrogen levels. The appropriate methods to reliably measure hormones in premenopausal women and the key biochemical isoforms relevant to breast cancer risk remain, however, unclear. Although the E1G measurement used in the study is novel, it is not without interpretative difficulties due to issues in measurement reproducibility and its unknown relationship(s) with hormone markers used in other studies; 3) a third potential limitation in understanding the role of sex steroids in cancer is related to using serum hormonal levels frequently to gauge the hormonal levels at the tissue level. This is especially problematic when using serum testosterone levels to study androgenic effects because the circulating testosterone is tightly bound to SHBG, while only the free hormone is bioactive [14]. SHBG and thus, total testosterone levels, vary widely based on genetic, metabolic, and endocrine influences. It is now suggested that measurement of free or bioavailable testosterone might predict androgenic effects more accurately than total testosterone levels [14]

\section{Sex Steroids and Breast Cancer Treatment}

In the first half of the twenty century, endocrine (also known as hormonal) therapy which involves reducing the amount of estrogen in the body or blocking the effect of estrogen became recognized as a viable treatment for breast cancer. This process depends on the presence of the hormone receptors: the estrogen and progesterone receptors (ER and PR). All breast tumors can be simplistically divided into hormone-receptor-positive $(\mathrm{HR}+)$ and hormone-receptor-negative (HR-) cases. Hormonal therapy is considered the main systemic treatment for $\mathrm{HR}+$ breast cancers and is not effective against HR- breast cancer. About $70 \%-80 \%$ of breast cancers are ER+. Almost $65 \%$ of ER+ breast cancers are also PR + . Nearly $13 \%$ cases are ER+ and PR- [18]. There are currently four main different modalities of hormonal therapies [18, 19]: 1) ovarian suppression or ablation using irreversible oophorectomy, irradiation, or more commonly the utilization of luteinizing hormone-releasing hormone (LHRH) analogs, which effects a temporary loss of ovarian function; 2) selective estrogen receptor modulators (SERM) (e.g., tamoxifen, toremifene); 3) aromatase inhibitors (anastrozole, exemestane, letrozole); and 4) estrogen receptors downregulators (fulvestrant). The first three modalities are used to the treatment of early stages of breast cancer while all four modalities can be used for the treatment for advanced metastatic breast cancer. The currently available aromatase inhibitors are anastrozole, exemestane, and letrozole.

Tamoxifen is the oldest and most-prescribed SERM, which binds to and inhibits estrogen receptor signaling in the breast. As a receptor antagonist, it is effective in both premenopausal and postmenopausal women. It has ER-stimulating effects in other tissues, including bone (resulting in preservation of bone density) and endometrium (leading to a 2- to 4-fold increased risk of endometrial cancer) [20,21]. Tamoxifen has been approved for breast cancer treatment since the early 1980s. It has been shown in multiple studies to decrease breast cancer associated mortality and recurrence versus placebo in the adjuvant treatment of breast cancer; 5 years of tamoxifen therapy resulting in about $50 \%$ reduction in recurrence and $25 \%$ reduction in mortality [22]. The common side effects associated with tamoxifen use include hot flashes (up to $80 \%)$, vaginal bleeding $(2 \%-25 \%)$ or discharge $(10 \%-55 \%)$, urinary frequency or urgency $(10 \%)$, mood changes $(15 \%-20 \%)$ or depression $(2 \%-12 \%)$, and thromboembolic events. For premenopausal women diagnosed with $\mathrm{HR}+$ breast cancer, tamoxifen remains the standard hormonal treatment [23]. Most recently, the result of the ATLAS (Adjuvant Tamoxifen Longer Against Shorter) trial was reported [24]. It showed that, among women with ER-positive disease, 10 years of tamoxifen (versus 5 years) reduced the risk of breast cancer recurrence (617 recurrences in 3428 women allocated to continuous tamoxifen versus 711 in 3418 controls, $\mathrm{p}=$ 0.002 ), reduced breast cancer mortality (331 deaths versus 397 deaths, $\mathrm{p}=0.01)$, and reduced overall mortality (639 deaths versus 722 deaths, $p=0 \cdot 01$ ). The reductions in adverse breast cancer outcomes appeared to be less extreme before than after year 10 (recurrence rate ratio [RR] 0.90 [95\% CI 0.79 - 1.02], during years 5 - 9, and 0.75 [0.62 - 0.90] in later years); breast cancer mortality 
0.97 [0.79 - 1.18] during years 5 - 9 and 0.71 [0.58 - 0.88] in later years. The cumulative risk of recurrence during years 5 - 14 was $21.4 \%$ for women allocated to continuous tamoxifen versus $25.1 \%$ for controls; breast cancer mortality during years $5-14$ was $12.2 \%$ for women allocated to continuous tamoxifen versus $15.0 \%$ for controls (absolute mortality reduction $2.8 \%$ ). This study has a major, immediate impact on premenopausal women with HR+ early breast cancer. Ongoing studies are attempting to clarify whether ovarian suppression is also necessary in premenopausal women diagnosed with $\mathrm{HR}+$ breast cancer as estrogens are mostly produced in ovaries [25]. This can be achieved using medication such as luteinizing-hormone-releasing hormone (LHRH) antagonist. e.g. goserelin, and leuprolide (temporary) or by surgically removing the ovaries (permanent suppression). For postmenopausal women, a number of studies have compared aromatase inhibitors with tamoxifen [26]. Aromatase is the enzyme (found in body fat, adrenal glands, and breast tissue as well as tumor cells) responsible for converting other steroid hormones into estrogen [26]. Aromatase is the sole source of estrogen in postmenopausal women. As the aromatase inhibitors have no effect on ovarian estrogen production, they are effective in postmenopausal women. Considering the overall results, it appears that an aromatase inhibitor is slightly superior in improving disease free survival in postmenopausal women with early stage $\mathrm{HR}+$ breast cancer with fewer serious side effects but with no improvement in overall survival compared to tamoxifen. Common side effects of AIs include hot flashes $(10 \%-35 \%)$, arthralgia/arthritis $(20 \%)$, headache $(10 \%-15 \%)$, vaginal dryness $(2 \%)$, and mood changes $(20 \%)$ [27,28]. Aromatase inhibitors are approved for the upfront adjuvant treatment of postmenopausal women with HR+ breast cancer. Other acceptable options include switching to an aromatase inhibitor after taking tamoxifen for 2 or 3 years (for a total of 5 years of hormonal therapy) as it has been shown to offer more benefits than 5 years of tamoxifen [30,31]. The optimal duration and sequence for the use of aromatase inhibitors has not been clearly defined, but their benefits for breast cancer recurrence and survival clearly support their use in all postmenopausal women [19]. Ongoing trials are evaluating outcomes in patients receiving longer durations of AI therapy. The MA.17R trial is evaluating letrozole versus placebo for 5 years in postmenopausal women with primary breast cancer who have completed about 5 years of AI therapy [32]. The National Surgical Adjuvant Breast and Bowel Project (NSABP) B-42 trial is evaluating 5 years of letrozole versus placebo in post-menopausal women with hormone recaptor-positive breast cancer who have completed 5 years of hormonal therapy (either 5 years of an AI or 5 years of a combination of up to 3 years of tamoxifen followed by an AI) [33]. Both trials are evaluating Disease Free Survival (DFS), overall survival, distant recurrence, and long-term safety. Androgens have a predominantly inhibitory effect on the growth of breast cancer cells, both in vitro and in vivo [34-37], potentially through induction of apoptosis [37]. Historically, androgens have been used successfully as hormonal therapy for advanced ER positive breast cancer [38]. However androgen therapy (e.g., fluoxymesterone or testosterone) has not gained popularity due to a high incidence of undesirable, virilizing side effects (Table 1).

\section{Conclusion}

Sex steroids are crucial hormones for the proper development and function of the body. They also play an important role in many common cancers, including breast cancer. This led to adopting endocrine manipulation as an effective methodology to treat many hormonally related cancers. Future studies are needed to better refine the optimal methods for measuring sex hormones and to better understand interrelationships of endogenous hormones with other circulating markers associated with cancer. In HR+ positive breast cancer, hormonal therapy is the mainstay of treatment. Hormonal treatments function stimulates existing micro-metastases or dormant cancer cells by decreasing estrogen's ability. The most commonly used types of hormonal therapy medicines include: 1) aromatase inhibitors (anastrozole, examestane, letrozole), for postmenopausal women with early or advanced breast cancer; 2) the selective estrogen receptor modulators (SERM) tamoxifen, used in pre- or postmenopausal women with early or advanced breast cancer; and 3 ) the estrogen receptors downregulator fulvestrant,

Table 1. Evolution of therapeutic hormonal therapy methods in breast cancer treatment.

\begin{tabular}{|c|c|c|}
\hline $\begin{array}{l}\text { Class/Hormonal } \\
\text { method }\end{array}$ & Side effects & Years \\
\hline & & $1950-1970 \mathrm{~s}$ \\
\hline Progesterone & \multicolumn{2}{|l|}{ Thromboembolism } \\
\hline Estrogen & \multicolumn{2}{|l|}{ Uterine cancer, cardiovascular disease } \\
\hline Prednisone & \multicolumn{2}{|l|}{ Hyperglycemia } \\
\hline Aminoglutethimide & \multicolumn{2}{|l|}{ Hepatotoxicity, hypothyroidism } \\
\hline Ovarian irrdiation & & \\
\hline Adnexectomy & \multicolumn{2}{|l|}{ Hirsutism, acne } \\
\hline \multicolumn{3}{|l|}{ Testosterone } \\
\hline & & $1980 \mathrm{~s}$ \\
\hline \multirow[t]{2}{*}{ Tamoxifen } & \multicolumn{2}{|c|}{ thromboembolic events, endometrial cancer } \\
\hline & & $1990 \mathrm{~s}$ \\
\hline Aromatase inhibitors & $\begin{array}{l}\text { Arthralgias, bone demineralization, } \\
\text { hypercholestrolemia }\end{array}$ & \\
\hline
\end{tabular}


used in postmenopausal women with advanced breast cancer. To date, there are no robust clinically available tools that can be used to reliably identify patients that are likely to be selectively responsive to one hormonal therapy versus another. Ongoing trials are attempting to identify the optimal duration of AI therapy. Tamoxifen given for 10 years appear to reduce recurrences and mortality in early stage breast cancer, based on findings from the ATLAS trial which might soon have an impact on the current standard of care for women taking tamoxifen.

\section{REFERENCES}

[1] C. G. Brook, "Mechanism of Puberty," Hormone Research, Vol. 51, Suppl. 3, 1999, pp. 52-54. doi: $10.1159 / 000053162$

[2] K. Svechnikov and O. Soder, "Ontogeny of Gonadal Sex Steroids," Best Practice \& Research Clinical Endocrinology \& Metabolism, Vol. 22, No. 1, 2008, pp. 95-106. doi:10.1016/j.beem.2007.09.002

[3] Z. Hyde, L. Flicker, K. A. McCaul, O. P. Almeida, G. J. Hankey, S. A. Chubb, et al., "Associations between Testosterone Levels and Incident Prostate, Lung, and Colorectal Cancer. A Population-Based Study," Cancer Epidemiology, Biomarkers \& Prevention, Vol. 21, No. 8, 2012, pp. 1319-1329. doi:10.1158/1055-9965.EPI-12-0129

[4] S. E. Hankinson, G. A. Colditz and W. C. Willett, "Towards an Integrated Model for Breast Cancer Etiology: the Lifelong Interplay of Genes, Lifestyle, and Hormones," Breast Cancer Research, Vol. 6, No. 5, 2004, pp. 213-218. doi:10.1186/bcr921

[5] J. D. Figueroa and L. A. Brinton, "Unraveling Genes, Hormones, and Breast Cancer," Journal of the National Cancer Institute, Vol. 104, No. 9, 2012, pp. 641-642. doi:10.1093/jnci/djs193

[6] P. H. Gann, C. H. Hennekens, J. Ma, C. Longcope and M. J. Stampfer, "Prospective Study of Sex Hormone Levels and Risk of Prostate Cancer," Journal of the National Cancer Institute, Vol. 88, No. 16, 1996, pp. 1118-1126. doi:10.1093/jnci/88.16.1118

[7] T. Key, P. Appleby, I. Barnes and G. Reeves, "Endogenous Sex Hormones and Breast Cancer in Postmenopausal Women: Reanalysis of Nine Prospective Studies," Journal of the National Cancer Institute, Vol. 94, No. 8, 2002, pp. 606-616. doi:10.1093/jnci/94.8.606

[8] S. A. Missmer, A. H. Eliassen, R. L. Barbieri and S. E. Hankinson, "Endogenous Estrogen, Androgen, and Progesterone Concentrations and Breast Cancer Risk among Postmenopausal Women," Journal of the National Cancer Institute, Vol. 96, No. 24, 2004, pp. 1856-1865. doi:10.1093/jnci/djh336

[9] R. Kaaks, S. Rinaldi, T. J. Key, F. Berrino, P. H. Peeters, C. Biessy, et al., "Postmenopausal Serum Androgens, Oestrogens and Breast Cancer Risk: The European Prospective Investigation into Cancer and Nutrition," Endocrine-Related Cancer, Vol. 12, No. 4, 2005, pp. 1071-
1082. doi: $10.1677 /$ erc. 1.01038

[10] T. J. Key, P. N. Appleby, G. K. Reeves, A. Roddam, J. F. Dorgan, C. Longcope, et al., "Body Mass Index, Serum Sex Hormones, and Breast Cancer Risk in Postmenopausal Women," Journal of the National Cancer Institute, Vol. 95, No. 16, 2003, pp. 1218-1226. doi:10.1093/jnci/djg022

[11] S. Rinaldi, T. J. Key, P. H. Peeters, P. H. Lahmann, A. Lukanova, L. Dossus, et al., "Anthropometric Measures, Endogenous Sex Steroids and Breast Cancer Risk in Postmenopausal Women: A Study within the EPIC Cohort," International Journal of Cancer, Vol. 118, No. 11, 2006, pp. 2832-2839. doi:10.1002/ijc. 21730

[12] Z. Nahleh and N. Tageja, "DHEA, Androgen Receptors, and Their Potential Role in Breast Cancer," DHEA in Human Health and Aging, Ed., Taylor \& Francis, Boca Raton, 2012.

[13] H. G. Burger, E. C. Dudley, J. Cui, L. Dennerstein and J. L. Hopper, "A Prospective Longitudinal Study of Serum Testosterone, Dehydroepiandrosterone Sulfate, and Sex Hormone-Binding Globulin Levels through the Menopause Transition," Journal of Clinical Endocrinology and Metabolism, Vol. 85, No. 8, 2000, pp. 2832-2838. doi:10.1210/jc.85.8.2832

[14] A. Vermeulen, L. Verdonck and M. Kaufman, "A Critical Evaluation of Simple Methods for the Estimation of Free Testosterone in Serum," Journal of Clinical Endocrinology and Metabolism, Vol. 84, No. 10, 1999, pp. 36663672. doi:10.1210/jc.84.10.3666

[15] X. Xu, T. D. Veenstra, S. D. Fox, J. M. Roman, H. J. Issaq, R. Falk, et al., "Measuring Fifteen Endogenous Estrogens Simultaneously in Human Urine by High-Performance Liquid Chromatography-Mass Spectrometry," Analytical Chemistry, Vol. 77, No. 20, 2005, pp. 66466654. doi: $10.1021 / \mathrm{ac} 050697 \mathrm{c}$

[16] K. Walker, O. Fletcher, N. Johnson, B. Coupland, V. A. McCormack, E. Folkerd, et al., "Premenopausal Mammographic Density in Relation to Cyclic Variations in Endogenous Sex Hormone Levels, Prolactin, and Insulin-Like Growth Factors," Cancer Research, Vol. 69, No. 16, 2009, pp. 6490-6499. doi:10.1158/0008-5472.CAN-09-0280

[17] N. Johnson, K. Walker, L. J. Gibson, N. Orr, E. Folkerd, B. Haynes, et al., "CYP3A Variation, Premenopausal Estrone Levels, and Breast Cancer Risk," Journal of the National Cancer Institute, Vol. 104, No. 9, 2012, pp. 657 669. doi:10.1093/jnci/djs156

[18] E. Winer, M. Morrow, K. Osborne, et al., "Cancer of the Breast," In: V. T. Devita, S. Hellman and S. A. Rosenberg, Eds., Principles and Practice of Oncology, 6th Edition, J.B. Lippincott Co., Philadelphia, 2001, pp. 1651-1717

[19] H. J. Burstein, A. A. Prestrud, J. Seidenfeld, H. Anderson, T. A. Buchholz, N. E. Davidson, et al., "American Society of Clinical Oncology Clinical Practice Guideline: Update on Adjuvant Endocrine Therapy for Women with Hormone Receptor-Positive Breast Cancer," Journal of Clinical Oncology, Vol. 28, No. 23, 2010, pp. 3784-3796. doi:10.1200/JCO.2009.26.3756

[20] R. C. Heel, R. N. Brogden, T. M. Speight and G. S. Avery, 
"Tamoxifen: A Review of Its Pharmacological Properties and Therapeutic Use in the Treatment of Breast Cancer," Drugs, Vol. 16, No. 1, 1978, pp. 1-24. doi:10.2165/00003495-197816010-00001

[21] "Effects of Adjuvant Tamoxifen and of Cytotoxic Therapy on Mortality in Early Breast Cancer. An Overview of 61 Randomized Trials among 28,896 Women. Early Breast Cancer Trialists' Collaborative Group," New England Journal of Medicine, Vol. 319, No. 26, 1988, pp. 1681-1692. doi:10.1056/NEJM198812293192601

[22] Early Breast Cancer Trialists' Collaborative Group, “Effects of Chemotherapy and Hormonal Therapy for Early Breast Cancer on Recurrence and 15-Year Survival: An Overview of the Randomized Trials," Lancet, Vol. 365, No. 9472, 2005, pp. 1687-1717. doi:10.1016/S0140-6736(05)66544-0

[23] P. Eifel, J. A. Axelson, J. Costa, J. Crowley, W. J. Curran, Jr., A. Deshler, et al., "National Institutes of Health Consensus Development Conference Statement: Adjuvant Therapy for Breast Cancer, November 1-3, 2000," Journal of the National Cancer Institute, Vol. 93, No. 13, 2001, pp. 979-989. doi:10.1093/jnci/93.13.979

[24] C. Davies, H. Pan, J. Godwin, R. Gray, R. Arriagada, V. Raina, et al., "Long-term Effects of Continuing Adjuvant Tamoxifen to 10 Years versus Stopping at 5 Years after Diagnosis of Oestrogen Receptor-Positive Breast Cancer: ATLAS, a Randomised Trial," Lancet, Vol. 381, No. 9869, 2012, pp. 805-816.

[25] "Suppression of Ovarian Function Plus Either Tamoxifen or Exemestane Compared with Tamoxifen Alone in Treating Premenopausal Women with Hormone-Responsive Breast Cancer (SOFT)." http://clinicaltrials.gov/show/NCT00066690

[26] M. Dowsett, J. Cuzick, J. Ingle, A. Coates, J. Forbes, J. Bliss, et al., "Meta-Analysis of Breast Cancer Outcomes in Adjuvant Trials of Aromatase Inhibitors versus Tamoxifen," Journal of Clinical Oncology, Vol. 28, No. 3, 2010, pp. 509-518. doi:10.1200/JCO.2009.23.1274

[27] S. R. Johnston and M. Dowsett, "Aromatase Inhibitors for Breast Cancer: Lessons from the Laboratory," Nature Reviews Cancer, Vol. 3, No. 11, 2003, pp. 821-831. doi:10.1038/nrc1211

[28] J. F. Forbes, J. Cuzick, A. Buzdar, A. Howell, J. S. Tobias and M. Baum, "Effect of Anastrozole and Tamoxifen as Adjuvant Treatment for Early-Stage Breast Cancer: 100-Month Analysis of the ATAC Trial," Lancet Oncology, Vol. 9, No. 1, 2008, pp. 45-53. doi:10.1016/S1470-2045(07)70385-6

[29] B. Thurlimann, A. Keshaviah, A. S. Coates, H. Mouridsen, L. Mauriac, J. F. Forbes, et al., "A Comparison of Letrozole and Tamoxifen in Postmenopausal Women with Early Breast Cancer," The New England Journal of
Medicine, Vol. 353, No. 26, 2005, pp. 2747-2757. doi:10.1056/NEJMoa052258

[30] M. Kaufmann, W. Jonat, J. Hilfrich, H. Eidtmann, G. Gademann, I. Zuna, et al., "Improved Overall Survival in Postmenopausal Women with Early Breast Cancer after Anastrozole Initiated after Treatment with Tamoxifen Compared with Continued Tamoxifen: The ARNO 95 Study," Journal of Clinical Oncology, Vol. 25, No. 19 2007, pp. 2664-2670. doi:10.1200/JCO.2006.08.8054

[31] R. C. Coombes, L. S. Kilburn, C. F. Snowdon, R. Paridaens, R. E. Coleman, S. E. Jones, et al., "Survival and Safety of Exemestane Versus Tamoxifen after 2 - 3 Years' Tamoxifen Treatment (Intergroup Exemestane Study): A Randomised Controlled Trial," Lancet, Vol. 369, No. 9561, 2007, pp. 559-570. doi:10.1016/S0140-6736(07)60200-1

[32] National Institutes of Health, "Letrozole in Treating Women with Primary Breast Cancer Who Have Received 5 Years of Aromatase Inhibitor Therapy," 2010. http://clinicaltrials.gov/ct2/show/NCT00754845_US

[33] National Institutes of Health, "Letrozole in Treating Women Who Have Received Hormone Therapy for Hormone Receptor-Positive Breast Cancer," 2010. http://clinicaltrials.gov/show/NCT00382070_US

[34] Y. de Launoit, S. Dauvois, M. Dufour, J. Simard and F. Labrie, "Inhibition of Cell Cycle Kinetics and Proliferation by the Androgen 5 Alpha-Dihydrotestosterone and Antiestrogen N,n-butyl-N-methyl-11-[16' alpha-chloro-3', 17beta-dihydroxy-estra-1',3',5'-(10')triene-7'alpha-yl] Undecanamide in Human Breast Cancer ZR-75-1 Cells," Cancer Research, Vol. 51, No. 11, 1991, pp. 2797-2802.

[35] M. A. Greeve, R. K. Allan, J. M. Harvey, J. M. Bentel, "Inhibition of MCF-7 Breast Cancer Cell Proliferation by 5alpha-Dihydrotestosterone; A Role for p21 (Cip1/Waf1)," Journal of Molecular Endocrinology, Vol. 32, No. 3, 2004, pp. 793-810. doi:10.1677/jme.0.0320793

[36] G. S. Gordan, A. Halden, Y. Horn, J. J. Fuery, R. J. Parsons and R. M. Walter, "Calusterone (7beta, 17alphaDimethyltestosterone) as Primary and Secondary Therapy of Advanced Breast Cancer," Oncology, Vol. 28, No. 2, 1973, pp. 138-146. doi:10.1159/000224811

[37] M. Kandouz, A. Lombet, J. Y. Perrot, D. Jacob, S. Carvajal, A. Kazem, et al., "Proapoptotic Effects of Antiestrogens, Progestins and Androgen in Breast Cancer Cells," Journal of Steroid Biochemistry and Molecular Biology, Vol. 69, No. 1-6, 1999, pp. 463-471.

[38] I. S. Goldenberg, N. Waters, R. S. Ravdin, F. J. Ansfield and A. Segaloff, "Androgenic Therapy for Advanced Breast cancer in Women. A Report of the Cooperative Breast Cancer Group," Journal of the American Medical Association, Vol. 223, No. 11, 1973, pp. 1267-1268. doi:10.1001/jama.1973.03220110045012 